Check for updates

Cite this: RSC Adv., 2019, 9, 2143

\title{
First-principles study of the electric, magnetic, and orbital structure in perovskite $\mathrm{ScMnO}_{3} \dagger$
}

\author{
Guang Song, (D)*ab Yuting Chen, ${ }^{a}$ Guannan $\mathrm{Li}^{\mathrm{ac}}$ and Benling Gao ${ }^{\mathrm{a}}$
}

Perovskite $\mathrm{SCMnO}_{3}$ has been synthesized under high temperature and high pressure. The magnetic ordering of this compound was proposed to be in the E-AFM state in previous theoretical research. Such magnetic ordering would lead the $\mathrm{Mn}^{3+}$ ions to be off-centered in the $\mathrm{MnO}_{6}$ octahedra; however, this is not detected experimentally. To address this issue, we systematically investigate the magnetic, orbital, and electric structures of perovskite $\mathrm{ScMnO}_{3}$ with first-principles calculations. It is found that its magnetic ground state is G-AFM and the magnetic ordering can explain the puzzle very well. Moreover, there is an unreported three-dimensional alternating cooperative orbital ordering in perovskite $\mathrm{ScMnO}_{3}$. The antiferromagnetic coupling between the nearest-neighbor $\mathrm{Mn}^{3+}$ ions is stabilized by the strong octahedral distortions that decrease the ferromagnetic interaction between the $\mathrm{e}_{\mathrm{g}}$ orbitals of the $\mathrm{Mn}^{3+}$ ions. In addition, we find that perovskite $\mathrm{ScMnO}_{3}$ is a bipolar antiferromagnetic semiconductor in which completely spin-polarized currents with reversible spin polarization can be tuned simply by applying a gate voltage. Such controllability of the spin polarization of the current opens up new avenues for future spintronic devices. Our results not only suggest that the G-AFM phase is the ground magnetic state for perovskite $\mathrm{SCMnO}_{3}$, but also enrich research in orbital ordering in rare-earth manganites.

Received 15th October 2018 Accepted 7th January 2019

DOI: $10.1039 / \mathrm{c} 8 \mathrm{ra0} 8507 \mathrm{~b}$

rsc.li/rsc-advances
Dy. ${ }^{\mathbf{8}, 21-24}$ Starting at Ho, the metastable orthorhombic $\mathrm{RMnO}_{3}$ systems are proposed to be E-AFM insulators. ${ }^{\mathbf{8 , 1 4 , 2 5}}$

Recently, perovskite $\mathrm{ScMnO}_{3}$ (PSMO) has been synthesized under high temperature and high pressure. ${ }^{26,27}$ Since the $\mathrm{Sc}^{3+}$ ion has the smallest radius among rare-earth elements, PSMO has a highly distorted structure and crystallizes into the monoclinic $P 2_{1} / n$ (space group, no. 14) phase. In this phase, there are two kinds of Mn atoms denoted as Mn1 and Mn2 (see Fig. 1). Additionally, there is a small deviation in $\beta$ (experimental value: $93.566^{\circ}$, see ref. 26 ) compared with other orthorhombic rare-earth manganites. Such highly distorted structures can be well understood from the Goldschmidt tolerance factor $\tau=\frac{R_{\mathrm{A}}+R_{\mathrm{O}}}{\sqrt{2\left(R_{\mathrm{B}}+R_{\mathrm{O}}\right)}}$, where $R_{\mathrm{A}}, R_{\mathrm{B}}$, and $R_{\mathrm{O}}$ are the radii of ions on the $\mathrm{A}, \mathrm{B}$, and $\mathrm{X}$ sites, respectively. ${ }^{28}$ In PSMO, the calculated $\tau$ is $0.83\left(\mathrm{LaMnO}_{3}: 0.902 ; \mathrm{LuMnO}_{3}: 0.840\right){ }^{29}$ indicating that PSMO has a larger lattice distortion than $\mathrm{LuMnO}_{3}$. In PSMO, the rotations and tilts of the $\mathrm{MnO}_{6}$ octahedra are the same as those of other rare-earth manganites, which can be expressed as $\mathrm{a}^{+} \mathrm{b}^{+} \mathrm{c}^{-}$in Glazer notation. However, the $\mathrm{Mn}-\mathrm{O}-\mathrm{Mn}$ bond angle in PSMO is quite different from those of other orthorhombic rare-earth manganites (see Fig. 2). Although the crystal structure of PSMO has been definitely confirmed in previous experimental studies, ${ }^{26,27}$ its ground magnetic state is still uncertain. Recently, in order to understand the effect of magnetic ordering on the distortion, a theoretical study based on density functional theory (DFT) has shown that the E-AFM phase has a lower energy than that of A-AFM. ${ }^{27}$ Moreover, it is
${ }^{a}$ Department of Physics, Huaiyin Institute of Technology, Huaian 223003, China ${ }^{b}$ Jiangsu Provincial Key Laboratory of Palygorskite Science and Applied Technology, Huaiyin Institute of Technology, Huaian 223003, China

${ }^{c}$ National Laboratory of Solid State Microstructures and Department of Physics, Nanjing University, Nanjing 210093, China.E-mail: gsong@hyit.edu.cn

$\dagger$ Electronic supplementary information (ESI) available. See DOI: 10.1039/c8ra08507b 

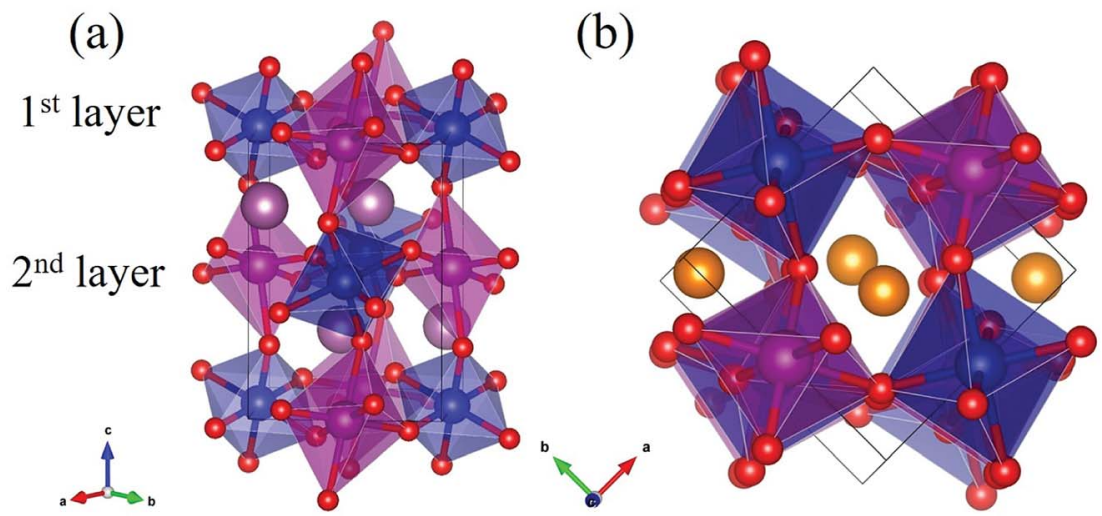

Fig. 1 The crystal structure of monoclinic $P 2_{1} / n \mathrm{ScMnO}_{3}$. (a) Top view: the labeled $1^{\text {st }}$ layer and $2^{\text {nd }}$ layer to distinguish the different $\mathrm{MnO} 6$ octahedra. (b) Side view. The positions of the unique atomic sites are labeled for clarity and the unique $\mathrm{MnO}_{6}$ octahedra are shaded magenta (Mn1) and blue (Mn2). In the figure, the golden and red spheres are Sc and O atoms, respectively.

also predicted that there are displacements of the Mn sites of $\sim 0.07 \AA$ in the E-AFM phase. However, such displacements are not detected by experimental study even at low temperature, ${ }^{27}$ indicating that the E-AFM phase cannot explain the experimental observation. Therefore, the magnetic ground ordering of PSMO still calls for more accurate theoretical models to

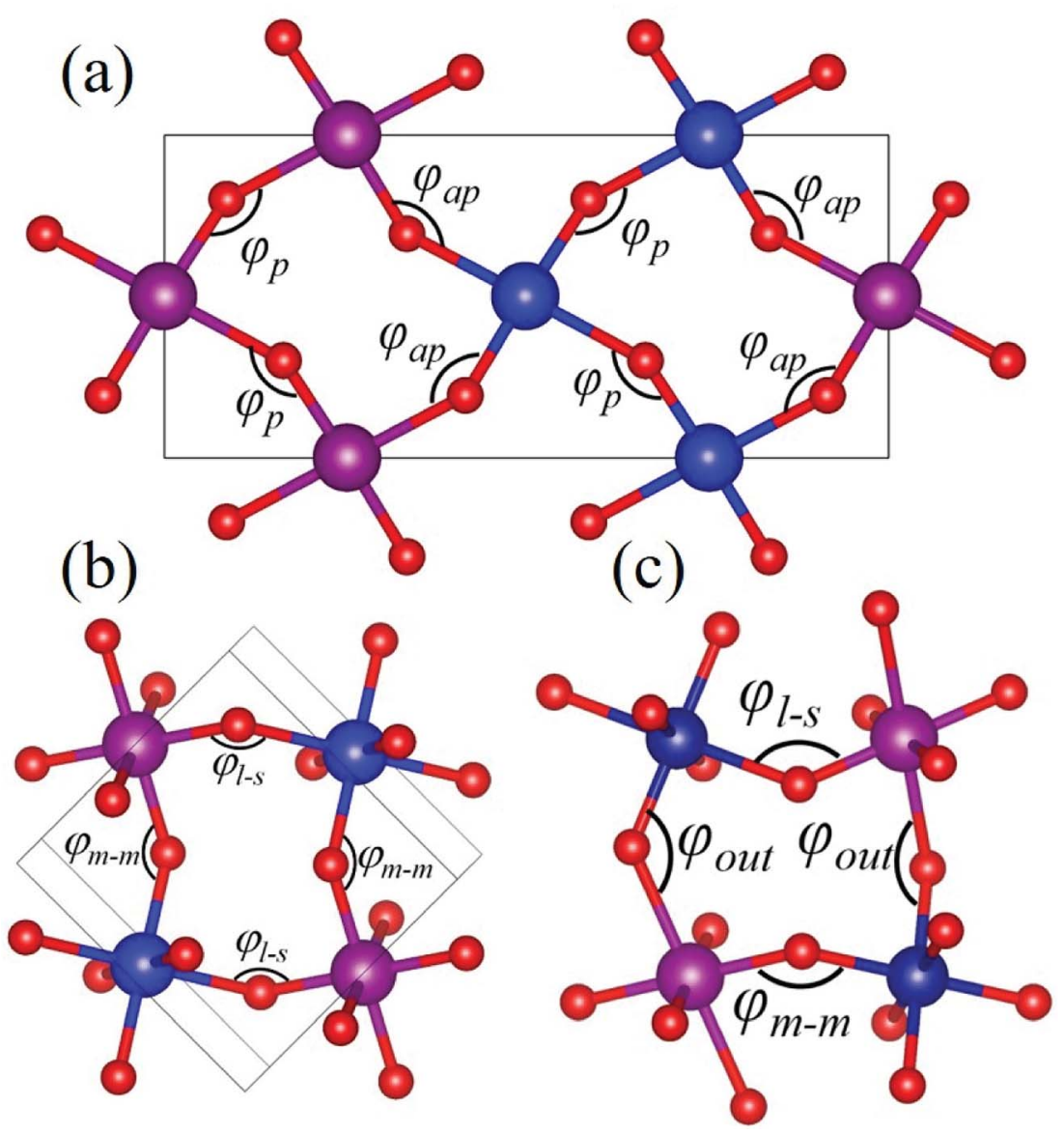

Fig. 2 Schematic diagrams of the $\mathrm{Mn}-\mathrm{O}-\mathrm{Mn}$ bond angle. The magenta and blue balls represent the spin-up and spin-down $\mathrm{Mn}^{3+}$ ions. (a) The bond angles in $\mathrm{HoMnO}_{3}$ and $\mathrm{LuMnO}_{3}$ with E-AFM magnetic ordering. The $\varphi_{\mathrm{ap}}$ is the bond angle between antiparallel-coupled Mn-spins; $\varphi_{\mathrm{p}}$ is the bond angle between parallel-coupled $\mathrm{Mn}$-spins. (b) The bond angle in $\mathrm{ScMnO}_{3}$ with $\mathrm{G}-\mathrm{AFM}$ magnetic ordering. The $\varphi_{l-s}$ is the bond angle composed by the long and short bond lengths; the $\varphi_{m-m}$ is the bond angle composed by the two middle bond lengths; the $\varphi_{\text {out }}$ is the bond angle along the c-axis. The magenta and blue balls represent spin-up and spin-down $\mathrm{Mn}$ ions, respectively. 
account for the controversy in such a highly perovskite compound.

The focus of the present work is to clarify the magnetic ground state of monoclinic $P 2_{1} / n \mathrm{ScMnO}_{3}$, in more general terms, to investigate its electric and orbital properties in depth. With first-principles calculations, we consider different magnetic alignments for the highly distorted manganite (FM, CAFM, A-AFM, G-AFM and E-AFM, see ESI Fig. S1†) $\mathrm{ScMnO}_{3}$. It is found that the G-AFM state is the magnetic ground state for PSMO and that such a state can give correct structural information in describing the displacements of $\mathrm{Mn}^{3+}$ ions. Moreover, we reveal a new type of orbital ordering which can be expressed as a $3 z^{2}-r^{2} / 3 y^{2}-r^{2}$-alternating manner in the $1^{\text {st }}$ layer and $3 x^{2}-r^{2} / 3 z^{2}-r^{2}$-alternating behavior in the $2^{\text {nd }}$ layer, and along the $c$ axis it forms $3 y^{2}-r^{2} / 3 z^{2}-r^{2}$-alternating and $3 z^{2}$ $-r^{2} / 3 x^{2}-r^{2}$-alternating orbital orderings in a chain-like manner in the two adjacent chains. In such a threedimensional staggered orbital ordering system, the competition between the antiferromagnetic and ferromagnetic interactions plays a relevant role in the stabilization of AFM interactions between nearest-neighbor $\mathrm{Mn}^{3+}$ ions. In addition, PSMO is identified as a bipolar antiferromagnetic semiconductor, in which completely spin-polarized currents with reversible spin polarization can be created and controlled simply by applying a gate voltage. Such controllability of the spin polarization of the current gives PSMO great potential applications in future spintronic devices. This study not only finds that the magnetic ground state of $\mathrm{ScMnO}_{3}$ is a G-AFM state, but also enlarges the field of orbital ordering in rareearth manganites.

\section{Computational methods}

First-principles calculations are carried out using DFT with projector augmented wave (PAW) potentials, ${ }^{30}$ as implemented in the Vienna ab initio simulation package (VASP). ${ }^{31,32}$ The PAW potentials explicitly include three valence electrons for Sc $\left(3 \mathrm{~d}^{1} 4 \mathrm{~s}^{2}\right), 13$ for $\mathrm{Mn}\left(2 \mathrm{p}^{6} 3 \mathrm{~d}^{5} 4 \mathrm{~s}^{2}\right)$, and 6 for $\mathrm{O}\left(2 \mathrm{~s}^{2} 2 \mathrm{p}^{4}\right)$ atoms. The exchange correlation functions are represented within the generalized gradient approximation (GGA) by the PBE and PBEsol parameterizations. ${ }^{33,34}$ Meanwhile, the local density approximation exchange correlation function (LDA) represented by the Cz-Pz parameterization is also used in optimizing the structure of $\mathrm{ScMnO}_{3} \cdot{ }^{35}$ Additionally, the GGA $+U$ method is also employed to investigate the influence of $+U$ correction. The rotationally invariant approach introduced by Dudarev $e t$ al. is used to treat the Coulomb repulsion parameter $U{ }^{36}$ To investigate the effect of the $U$ value on the calculated results, we use $U$ values changing over the range $1-5 \mathrm{eV}$ with a fixed exchange parameter $J=1 \mathrm{eV}$ for the $3 \mathrm{~d}$ orbital of $\mathrm{Mn}$ ions. The wave function is expanded in a plane wave basis with an energy cutoff of $600 \mathrm{eV} .6 \times 6 \times 4 \Gamma$-centered $k$-point sampling is used for reciprocal space integrations in calculating the FM, C-AFM, AAFM, and G-AFM magnetic states (a $\sqrt{2} \times \sqrt{2} \times 2$ supercell is used in the calculations), and $6 \times 3 \times 4 \Gamma$-centered $k$-point sampling is used for the E-AFM magnetic state $(\mathrm{a} \sqrt{2} \times 2 \sqrt{2} \times 2$ supercell is used in the calculations). We have carefully checked convergence with these settings by testing higher energy cutoffs and larger $k$-point meshes. Each self-consistent electronic calculation is converged to $10^{-6} \mathrm{eV}$ and the tolerance force is converged to less than $1 \mathrm{meV}^{-1}$.

\section{Results and discussion}

The optimized lattice parameters of PSMO with different magnetic orderings are summarized in Table 1 together with the experimental data $(293 \mathrm{~K}) .{ }^{26}$ The agreement between the calculated and experimental crystal parameters is perfect, and the relative errors are all within 3\% when the PBE and PBEsol functions are used in the calculations, while the relative error exceeds $8 \%$ when the $\mathrm{Cz}-\mathrm{Pz}$ function is used. The total energies for various possible magnetic orderings are calculated with PBE, PBEsol, and Cz-Pz parameterizations, and the calculated results are summarized in Table 1. It is found that the magnetic ordering of the ground state is G-AFM phase within the GGA method (including PBE and PBEsol functions), while the ground magnetic ordering is FM within the LDA method. It should be noted that the magnetic state of PSMO is proposed to be antiferromagnetic ordering experimentally. ${ }^{26}$ Thus the LDA method not only overly underestimates the lattice parameters, but also gives a dissimilar magnetic ordering compared with experimental data. Therefore, we do not use the LDA method in the next study and continue to use the GGA method to investigate the structure, electric, and orbital properties of $\mathrm{ScMnO}_{3}$.

Then we discuss whether the proposed G-AFM state can explain the structural distortions observed experimentally. To obtain the distortion of the $\mathrm{MnO}_{6}$ octahedra in PSMO, we calculate the bond lengths of $\mathrm{Mn}-\mathrm{O}$ of different magnetic orderings, and the results are listed in Table 2. Herein, it should be emphasized that the two calculated bond lengths are equal along the local axis of the $\mathrm{MnO}_{6}$ octahedra for the G-AFM, FM, $\mathrm{C}-\mathrm{AFM}$, and A-AFM phases; thus, the $\mathrm{Mn}^{3+}$ ions are in the center of the $\mathrm{MnO}_{6}$ octahedra. It is, therefore, indicated that there is no displacement of $\mathrm{Mn}^{3+}$ ions in PSMO with these magnetic orderings. Conversely, for the E-AFM phase, we can see that the bond lengths are different along the local axis of the $\mathrm{MnO}_{6}$ octahedra; thus, the $\mathrm{Mn}^{3+}$ ions are not in the center of the $\mathrm{MnO}_{6}$ octahedra any more. From the difference in bond lengths, one can calculate the displacements of $\mathrm{Mn}^{3+}$ ions. The calculated magnitudes of displacements away from the center are about 0.06 and $0.13 \AA$ for the PBEsol and PBE functions, respectively. In a previous DFT study, the calculated displacements of $\mathrm{Mn}^{3+}$ ions are about $0.07 \AA$ for the E-AFM phase. However, these displacements are not detected experimentally. ${ }^{27}$ Therefore, it is suggested that the ground magnetic ordering of PSMO is not EAFM. Based on the calculated total energies of PSMO with different magnetic orderings, one can conclude that the magnetic ground state is G-AFM. It should also be emphasized that the G-AFM state is a new magnetic ordering in the family of rare-earth manganites. ${ }^{15}$

Another interesting result is the distortions of $\mathrm{MnO}_{6}$ octahedra, which have a significant effect on the electric structures 
Table 1 The calculated lattice parameters and energy differences of PSMO with different magnetic orderings (in meV/Mn). The experimental values are taken from ref. 26 . The energy of the FM phase is chosen to be zero in calculating the energy difference

\begin{tabular}{|c|c|c|c|c|c|c|c|}
\hline & Exp. & Method & G-AFM & E-AFM & C-AFM & A-AFM & FM \\
\hline \multirow[t]{2}{*}{$a(\AA)$} & 5.053 & PBEsol & 4.965 & 4.967 & 4.955 & 4.991 & 4.985 \\
\hline & & PBE & 5.056 & 5.059 & 5.045 & 5.080 & 5.073 \\
\hline \multirow[t]{3}{*}{$b(\AA)$} & 5.355 & PBEsol & 5.335 & 5.353 & 5.329 & 5.363 & 5.360 \\
\hline & & PBE & 5.357 & 5.366 & 5.356 & 5.374 & 5.378 \\
\hline & & $\mathrm{Cz}-\mathrm{Pz}$ & 5.335 & 5.330 & 5.319 & 5.312 & 5.306 \\
\hline & & $\mathrm{Cz}-\mathrm{Pz}$ & 7.089 & 7.052 & 7.070 & 7.040 & 7.037 \\
\hline \multirow[t]{3}{*}{$\beta\left(^{\circ}\right)$} & 93.566 & PBEsol & 94.325 & 93.648 & 93.765 & 93.672 & 92.743 \\
\hline & & PBE & 94.702 & 94.785 & 94.993 & 94.780 & 94.468 \\
\hline & & $\mathrm{Cz}-\mathrm{Pz}$ & 90.088 & 90.082 & 90.085 & 90.088 & 90.075 \\
\hline \multirow[t]{2}{*}{$\Delta E(\mathrm{meV})$} & - & PBEsol & -63.2 & -53.7 & -55.0 & -15.0 & 0.0 \\
\hline & & PBE & -52.8 & -40.8 & -41.4 & -17.5 & 0.0 \\
\hline
\end{tabular}

Table 2 The calculated bond lengths of PSMO with different magnetic orderings (in A). The experimental values are taken from ref. 26

\begin{tabular}{llllllll}
\hline Bond & Exp. & Method & G-AFM & E-AFM & C-AFM & A-AFM & FM \\
\hline Mn1-O2 & \multirow{2}{*}{1.920} & PBEsol & 1.888 & $1.907,1.887$ & 1.909 & 1.897 & 1.932 \\
& & PBE & 1.902 & $1.915,1.895$ & 1.913 & 1.903 & 1.917 \\
Mn1-O3 & 1.967 & PBEsol & 1.942 & $1.936,1.922$ & 1.951 & 1.934 & 1.938 \\
& & PBE & 1.965 & $1.970,1.958$ & 1.968 & 1.969 & 1.969 \\
Mn1-O1 & 2.138 & PBEsol & 2.115 & $2.108,2.071$ & 2.093 & 2.102 & 2.075 \\
& & PBE & 2.202 & $2.208,2.170$ & 2.185 & 2.200 & 2.189 \\
Mn2-O1 & \multirow{2}{*}{1.902} & PBEsol & 1.871 & $1.891,1.875$ & 1.871 & 1.892 & 1.896 \\
& & PBE & 1.891 & $1.903,1.891$ & 1.889 & 1.906 & 1.905 \\
Mn2-O3 & 1.930 & PBEsol & 1.917 & $1.960,1.921$ & 1.914 & 1.947 & 1.953 \\
& & PBE & 1.925 & $1.948,1.922$ & 1.925 & 1.934 & 1.944 \\
Mn2-O2 & 2.320 & PBEsol & 2.283 & $2.250,2.205$ & 2.240 & 2.229 & 2.166 \\
& & PBE & 2.442 & $2.468,2.371$ & 2.413 & 2.422 & 2.385
\end{tabular}

of transition metal ions. The distortion can be described by two normal modes, $Q_{2}$ and $Q_{3}$, which are represented by $Q_{2}=\sqrt{2(l-s)}$, and $Q_{3}=\frac{2}{\sqrt{6}}(2 m-l-s)^{1,37}$, where $l=$ long bond length, $m=$ medium bond length, and $s=$ short bond length. Because of the two unique $\mathrm{Mn}^{3+}$ ions in PSMO, there are two $Q_{2}$ and two $Q_{3}$ modes in PSMO. The calculated results are

Table 3 The calculated normal modes $Q_{2}$ and $Q_{3}$ for PSMO with different magnetic orderings. When calculating the normal modes for the E-AFM phase, the $l, m$, and $s$ are averaged

\begin{tabular}{llllllll}
\hline & Exp. & Method & G-AFM & E-AFM & C-AFM & A-AFM & \multicolumn{1}{l}{ FM } \\
\hline \multirow{2}{*}{ Mn1- $Q_{2}$} & 0.308 & PBEsol & 0.321 & 0.272 & 0.260 & 0.290 & 0.202 \\
& 0.322 & PBE & 0.424 & 0.401 & 0.384 & 0.420 & 0.385 \\
Mn2- $Q_{2}$ & 0.591 & PBEsol & 0.591 & 0.488 & 0.521 & 0.477 & 0.382 \\
& 0.571 & PBE & 0.779 & 0.738 & 0.741 & 0.729 & 0.679 \\
Mn1- $Q_{3}$ & -0.101 & PBEsol & -0.097 & -0.105 & -0.082 & -0.107 & -0.107 \\
& -0.098 & PBE & -0.142 & -0.136 & -0.132 & -0.135 & -0.137 \\
Mn2-Q & -0.296 & PBEsol & -0.394 & -0.366 & -0.231 & -0.185 & -0.127 \\
& -0.270 & PBE & -0.261 & -0.187 & -0.369 & -0.376 & -0.329
\end{tabular}

summarized in Table 3. One can see that the calculated values of the distortion mode of $\mathrm{ScMnO}_{3}$ with the G-AFM phase within the PBEsol function is quite close to the experimental data. ${ }^{27}$ Additionally, the modes of Mn1 and Mn2 are not equal. It is indicated that the local environments of the nearest-neighbor (NN) Mn ions are distinct from each other. This difference may induce a charge transfer between NN Mn ions. The electron numbers of d-orbital electrons of $\mathrm{Mn}$ ions have been calculated with the PBEsol function and they are 4.95 and 4.98e for Mn1 and Mn2, respectively. Obviously, a weak charge ordering of NN Mn ions exists in PSMO. Herein, the fascinating thing is that the origin of this charge order is quite different from the $\mathrm{Mn}^{4+} / \mathrm{Mn}^{3+}$ charge order in $\mathrm{La}_{0.7} \mathrm{Ca}_{0.3} \mathrm{MnO}_{3}{ }^{38}$ in which the charge order is induced by doping the alkaline-earth metal, while in PSMO the charge transfer is just related to the distortion of the $\mathrm{MnO}_{6}$ octahedra.

Now let us discuss how the distortion of the $\mathrm{MnO}_{6}$ octahedra affects the electronic structure of PSMO. From the $\mathrm{Mn}-\mathrm{O}$ bond lengths listed in Table 2, one can clearly see that the $\mathrm{Mn}-\mathrm{O}$ bond in the $\mathrm{MnO}_{6}$ octahedron is elongated in one direction, while being compressed in another direction. It is well known that such a distortion will make the double degenerate $e_{g}$ orbital in an ideal cubic perovskite split into two nondegenerate energy levels: one is the $3 z^{2}-r^{2}$ orbital and the other is the $x^{2}-y^{2}$ orbital. The famous example is the distortion of $\mathrm{MnO}_{6}$ octahedra in $\mathrm{LaMnO}_{3}$, in which the $\mathrm{MnO}_{6}$ octahedra are alternately elongated in the $x y$ plane. Therefore, a $3 x^{2}-r^{2} / 3 y^{2}-r^{2}$ orbital ordering has been reported in $\mathrm{LaMnO}_{3}{ }^{18}$ We wondered whether such an ordering will appear in PSMO. After systematically analyzing the $\mathrm{Mn}-\mathrm{O}$ listed in Table 2, we find that in layer 1 the $\mathrm{MnO}_{6}$ octahedron at the Mn1 site is elongated along the $z$ axis, while the $\mathrm{MnO}_{6}$ octahedron at the $\mathrm{Mn} 2$ site is elongated along the $y$ axis. Thus, the orbital ordering for such a distortion can be expressed as $3 z^{2}-r^{2} / 3 y^{2}-r^{2}$ in layer 1. In layer 2, the $\mathrm{MnO}_{6}$ octahedra at the Mn2 sites are elongated along the $x$ axis, while the $\mathrm{MnO}_{6}$ octahedra at the Mn1 sites are elongated along the $y$ axis. Thus, in such a frame, the orbital ordering can be 

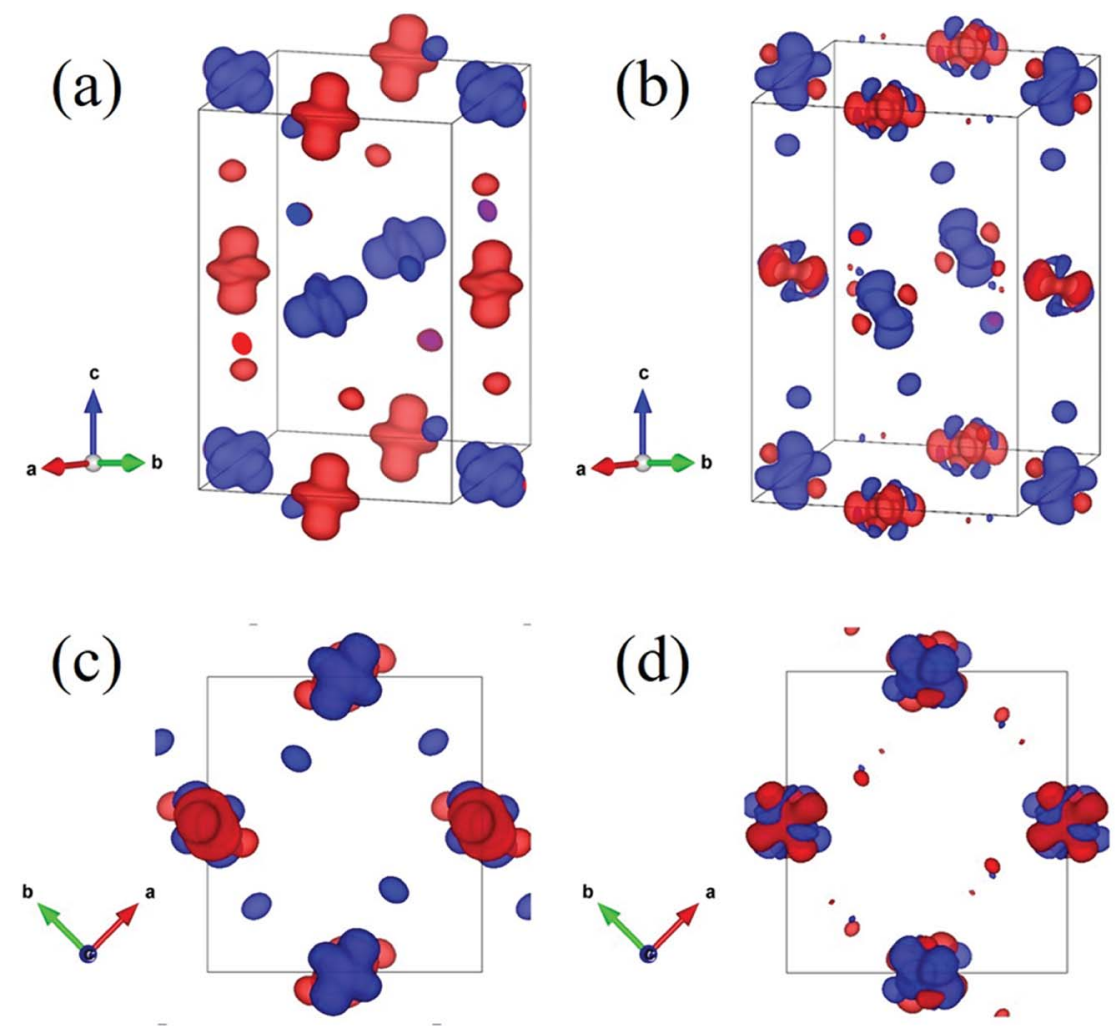

Fig. 3 The calculated $e_{\mathrm{g}}$ spin charge density distributions of the $P 2_{1} / n$ with the G-AFM state within the GGA $+U\left(U_{\text {eff }}=1.0\right.$ eV) method. The energy windows of $1.5 \mathrm{eV}$ widths just below the Fermi level are plotted in (a) and (c) which characterize the $3 z^{2}-r^{2}, 3 x^{2}-r^{2}$, and $3 y^{2}-r^{2}$ orbitals; the energy window of $1.5 \mathrm{eV}$ widths just above the Fermi level are plotted in (b) and (d) which characterize the $x^{2}-y^{2}, y^{2}-z^{2}$, and $z^{2}-x^{2}$ orbitals. The red and blue isosurfaces show spin-up and spin-down charge densities, respectively. (a) and (b) are side views; (c) and (d) are top views along the [001] direction.

expressed as $3 x^{2}-r^{2} / 3 z^{2}-r^{2}$ in layer 2. Additionally, along the $z$ axis they form $3 z^{2}-r^{2} / 3 x^{2}-r^{2}$-alternating and $3 y^{2}-r^{2} / 3 z^{2}-r^{2}$ alternating orbital orderings in a chain-like manner in the two adjacent chains. In order to characterize the orbital orderings more clearly, the spin charge density of the $e_{g}$ orbital is calculated within the PBEsol method. Fig. 3a and $\mathrm{c}$ show the $\mathrm{e}_{\mathrm{g}}$ spin charge density integrated from $-1.5 \mathrm{eV}$ to the Fermi level; Fig. $3 \mathrm{~b}$ and d plot the $\mathrm{e}_{\mathrm{g}}$ spin charge density integrated from the Fermi level to $1.5 \mathrm{eV}$. It can clearly be seen that the spin charge density of the Mn1 site has a predominantly $3 z^{2}-r^{2}$ orbital character, while at the $\mathrm{Mn} 2$ site the charge density has predominantly $3 y^{2}-r^{2}$ and $3 x^{2}-r^{2}$ orbital characters for layer 1 and layer 2, respectively. Therefore, such an alternating arrangement of orbital ordering in PSMO is three-dimensional and it is quite different from the in-plane two-dimensional orbital ordering $\left(3 x^{2}-r^{2} / 3 y^{2}-r^{2}\right.$ orbital ordering) in other $\mathrm{RMnO}_{3}(\mathrm{R}=\mathrm{La}-\mathrm{Lu}, \mathrm{Y}){ }^{9,14,16-18,23}$ It should also be emphasized that this three-dimensional orbital ordering is different from other three-dimensional orbital ordering, such as in strained $\mathrm{LaMnO}_{3}$ (where the behavior of the orbital ordering is expressed as $\left.3 z^{2}-r^{2} / x^{2}-y^{2}\right)^{18,39}$ and in bulk $\mathrm{YVO}_{3}$ (the $\mathrm{VO}_{6}$ octahedra are alternately elongated along the $x$ and $y$ axes in the $x y$ plane in the first layer and along the $y$ and $x$ axes in the $x y$ plane in the second layer; the behavior of the orbital ordering is expressed as $x z / y z$ in the first layer and $y z / x z$ in the second layer) (see
Fig. S2 $\uparrow) .^{40}$ Therefore, we reveal that PSMO possesses an unreported three-dimensional competitive orbital ordering. This result enriches research on orbital ordering in the family of rare-earth manganites.

Having systematically discussed the orbital ordering, now let us study how the three-dimensional cooperative orbital

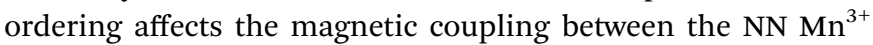
ions. In PSMO, the spin-spin coupling consists of antiferromagnetic interactions $\left(J^{\mathrm{A}}\right.$, from the $\mathrm{t}_{2 \mathrm{~g}}-\mathrm{O}-\mathrm{t}_{2 \mathrm{~g}}$ and $\mathrm{t}_{2 \mathrm{~g}}-\mathrm{O}-\mathrm{e}_{\mathrm{g}}$ orbitals) and ferromagnetic e-O-e interactions $\left(J^{\mathrm{F}}\right.$, from the $\mathrm{e}_{g}$ orbital), respectively. According to the Goodenough-Kanamori rules ${ }^{41,42}$ the magnetic interactions between $\mathrm{NN} \mathrm{Mn}^{3+}$ ions in monoclinic $\mathrm{ScMnO}_{3}$ should be $\mathrm{FM}$, as shown in the $a b$ plane of $\mathrm{LaMnO}_{3}$ in which the absolute value of $J^{\mathrm{F}}\left(J^{\mathrm{F}}<0\right)$ is stronger than that of $J^{\mathrm{A}}\left(J^{\mathrm{A}}>0\right) \cdot{ }^{17}$ However, our calculated results predict an AFM interaction between NN Mn-Mn ions. This seems to be in contradiction with the Goodenough-Kanamori rule. This discrepancy is because the $<\mathrm{Mn}-\mathrm{O}-\mathrm{Mn}$ bond angle $\varphi$ is much smaller than $180^{\circ}$ in perovskite monoclinic $\mathrm{ScMnO}_{3}$. As shown by a previous model and DFT studies, the bond angle $\varphi$ plays an important role in determining the magnetic interaction, through altering the relative magnitude of $J^{\mathrm{A}}$ and $J^{\mathrm{F}}$ interactions. ${ }^{15,16,43,44}$ It is well known that the hopping integral between Mn- $\mathrm{e}_{\mathrm{g}}$ states depends both on the bond angle $\varphi$ and on the bond length. The $t_{\mathrm{dp}}$ (the overlap integral between Mn-d and O- 

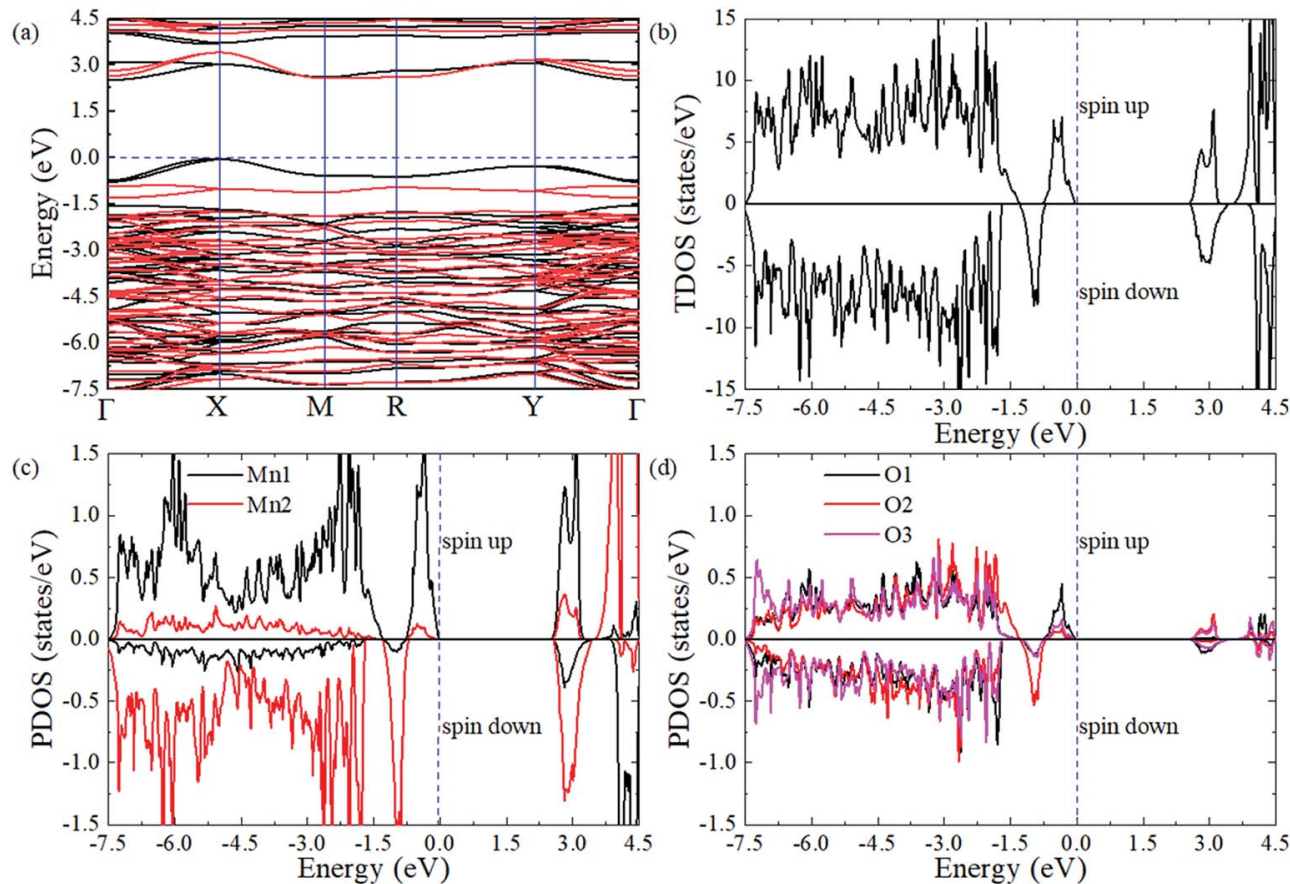

Fig. 4 The calculated electric properties for monoclinic PSMO in the G-AFM magnetic orderings calculated within the HSE06 function. (a) Band structure. The black and red lines represent the spin-up and spin-down states, respectively. (b) Total DOS. (c) Projected DOS for the Mn-d orbital. (d) Projected DOS for the O-p orbital. The blue dashed line represents the Fermi level and it is set as the zero of the energy scale.

p states) is roughly described by $t_{\mathrm{dp}} \propto \frac{\left[\frac{180^{\circ}-\varphi}{2}\right]}{d^{3.5}}$, where $d$ is the Mn-O bond length. ${ }^{16,45}$ According to the picture outlined by Zhou and Goodenough, the $J^{\mathrm{F}}$ interactions decrease with $\varphi$ while the $J^{\mathrm{A}}$ interactions are nearly constant. ${ }^{16}$ The continuous reduction of $J^{\mathrm{F}}$ in $J=J^{\mathrm{F}}+J^{\mathrm{A}}$ as $\varphi$ decreases could induce $J$ to change from negative to positive. Based on the Heisenberg Hamiltonian, Yamauchi et al. calculate the magnetic interactions in orthorhombic $\mathrm{RMnO}_{3}(\mathrm{R}=\mathrm{La}-\mathrm{Lu}, \mathrm{Y})$. They find that as $\mathrm{R}$ changes from $\mathrm{La}$ to $\mathrm{Lu}$, the $J$ monotonously increases from -12.9 to $0.2 \mathrm{meV}^{43}$ According to previous theoretical and experimental reports, the magnetic couplings between the NN $\mathrm{Mn}$ atoms are antiferromagnetic when the bond angles are less than $143.00^{\circ} .^{15}$

Herein, as a reference, we calculate the bond angles of perovskite $\mathrm{HoMnO}_{3}$ and $\mathrm{LuMnO}_{3}$ within the PBEsol method, because in these compounds, the in-plane magnetic couplings are both ferromagnetic and antiferromagnetic (see Fig. 2a). In $\mathrm{HoMnO}_{3}$, the calculated bond angles are $142.09^{\circ}$ and $145.89^{\circ}$ for $\varphi_{\mathrm{ap}}$ and $\varphi_{\mathrm{p}}$, respectively. In E-AFM $\mathrm{LuMnO}_{3}$, the calculated bond angles are $140.84^{\circ}$ and $144.60^{\circ}$ for $\varphi_{\text {ap }}$ and $\varphi_{\mathrm{p}}$, respectively. Due to the three-dimensional orbital ordering, the bond angles considered in PSMO not only include the in-plane angles $\left(\varphi_{m-m}\right.$ and $\varphi_{l-s}$, see Fig. $2 \mathrm{~b}$ ), but also include the out-of-plane angles $\left(\varphi_{\text {out }}\right.$, see Fig. $\left.2 \mathrm{c}\right)$. In order to study the effect of the orbital ordering on the magnetic properties of this system, we calculated bond angles in PSMO. Within the PBEsol method, the calculated in-plane bond angles are $132.10^{\circ}$ and $141.58^{\circ}$ for $\varphi_{m-}$ ${ }_{m}$ and $\varphi_{l-s}$, respectively. Obviously, the in-plane bond angles are both less than $143^{\circ}$; thus the in-plane magnetic interactions between the $\mathrm{NN} \mathrm{Mn}^{3+}$ ions are antiferromagnetic along both the $x$ and $y$ axes. Additionally, the calculated out-of-plane bond angle is $132.10^{\circ}$ (still less than $143^{\circ}$ ). Therefore, the magnetic interaction along the $z$ axis is also antiferromagnetic. To understand the microscopic origins of the G-AFM ground state PSMO, the exchange interactions are extracted from the calculated total energy of all considered magnetic orderings. Based on the Heisenberg Hamiltonian with a normalized spin moment used in a previous report, ${ }^{43}$ we can estimate the superexchange interaction energies $J_{i}(i=x, y, z)$. The calculated results are $6.0,2.8$, and $3.3 \mathrm{meV}$ for $J_{x}, J_{y}$, and $J_{z}$, respectively. The positive values indicate that the magnetic interactions are all antiferromagnetic.

To obtain a comprehensive understanding of PSMO, we study its electric properties with a hybrid Heyd-Scuseria-Ernzerhof function (HSE06, the standard exact-exchange mixing parameter $\alpha$ of 0.25 is used).$^{46}$ As shown in Fig. 4a, the valence band maximum (VBM) locates at the $X$ point while the conduction band minimum (CBM) locates at the $\Gamma$ point, suggesting that the PSMO is an indirect band gap semiconductor with a band gap of $2.50 \mathrm{eV}$ (the spin-down band gap is $2.57 \mathrm{eV}$ and the spin-up band gap is $2.50 \mathrm{eV}$ ). Interestingly, it is can be seen from the band structure (Fig. 4a) and the total density of states (DOS, Fig. 4b) that the spin-down bands and spin-up bands are not symmetrically occupied. Based on the above discussion, we can conclude that PSMO is a bipolar antiferromagnetic semiconductor, ${ }^{47}$ which can provide completely spinpolarized currents with tunable spin polarization simply by applying a gate voltage. In PSMO, when the Fermi energy is 
tuned in the range $-0.78-0 \mathrm{eV}$, the conducting channel is opened for spin-up electrons; when the Fermi energy is tuned in the range $-1.30 \sim-0.78 \mathrm{eV}$, the conducting channel is opened for spin-down electrons. Such controllability of the spin polarization of current opens up new avenues for future spintronic devices. We also plot the partial density of states projected onto the Mn-d and O-p orbitals to investigate the component in the conduction and valence band edges, as shown in Fig. 4c and d. The valence and conduction band edges are mainly contributed by the Mn-d orbital. It can be seen that the split energies (the energy difference between the $3 z^{2}-r^{2}$ and $x^{2}-y^{2}$ orbitals) induced by the octahedral distortions are 2.50 and $3.28 \mathrm{eV}$ for the Mn1 and Mn2 ions, respectively. Below the Fermi level the $[-2.1 \mathrm{eV}, 0 \mathrm{eV}]$ energy window is mainly occupied by the $3 z^{2}-r^{2}$ orbital of Mn ions, whereas above the Fermi level the $[2.50 \mathrm{eV}$, $3.45 \mathrm{eV}]$ energy window is mainly composed by the $x^{2}-y^{2}$ orbital of Mn ions. Moreover, in the $[-7.50 \mathrm{eV}, 3.16 \mathrm{eV}]$ energy window, the d orbital of $\mathrm{Mn}$ ions is strongly hybridized with the $\mathrm{p}$ orbital of $\mathrm{O}$ ions.

In the above calculations, we do not consider the electronic correlation of the d-electron of $\mathrm{Mn}^{3+}$ ions. It is generally accepted that the correlation has a notable effect on the magnetic ground state in rare-earth manganites..$^{\mathbf{4 3 4 , 4 8 - 5 1}}$ For instance, previous reports have pointed out that the small $U$ $(\sim 2.5 \mathrm{eV})$ is found to stabilize A-AFM with respect to E-AFM in distorted manganites such as $\mathrm{HoMnO}_{3}$ and $\mathrm{LuMnO}_{3}$ at variance with experiments, which show E-AFM as the ground state. Moreover, previous studies also showed that as the U parameter increases, the magnetic ordering will be FM. Additionally, with the constrained random-phase-approximation method, Solovyev obtained $U_{\text {eff }}=U-J=1.30 \mathrm{eV}(U=2.15 \mathrm{eV}$ and $J=0.85$ $\mathrm{eV}), U_{\text {eff }}=U-J=1.38 \mathrm{eV}(U=2.24 \mathrm{eV}$ and $J=0.86 \mathrm{eV})$, and $U_{\text {eff }}$ $=U-J=1.31 \mathrm{eV}(U=2.16 \mathrm{eV}$ and $J=0.85 \mathrm{eV})$ for the d orbitals of $\mathrm{Mn}^{3+}$ ions of orthorhombic $\mathrm{LaMnO}_{3}, \mathrm{TbMnO}_{3}$, and $\mathrm{HoMnO}_{3}$, respectively. ${ }^{52}$ This indicated that the magnetic ordering of ground state distorted manganites is rather strongly affected by the $U$ parameter, which is, unfortunately, experimentally not known for $\mathrm{ScMnO}_{3}$. Nevertheless, we also carried out DFT $+U$ calculations to investigate the influence of electronic correlation on the stability of the magnetic ground states. We recalculated the total energy of all spin orders using the GGA $+U$ method with $U_{\text {eff }}$ values changing over the range $U_{\text {eff }}=0-4 \mathrm{eV}$. The calculated results are displayed in Fig. 5 . It can clearly be seen that the G-AFM state is the ground state when $U_{\text {eff }}$ is less than $2.30 \mathrm{eV}$; then the E-AFM state is the ground state when $U_{\text {eff }}$ is between 2.30 and $2.70 \mathrm{eV}$; and the FM state is the ground state when $U_{\text {eff }}$ is larger than $2.70 \mathrm{eV}$. Obviously, we find a similar tendency in previous reports on the magnetic ordering being affected by the $U$ parameter. ${ }^{\mathbf{4 4 5 0 , 5 1}}$ This result can be attributed to the significant reduction in antiferromagnetic exchange interaction $\left(J^{\mathrm{A}}\right)$, since the $+U$ treatment enhances the localization of $t_{2 g}$ states. While the $e_{g}$ states are not directly affected by + $U$ treatment due to the strong hybridization between the $\mathrm{e}_{\mathrm{g}}$ and O-p states $\left(J^{\mathrm{F}}\right)$. Thus, it is suggested that the smaller electronic correlation can give the correct magnetic ground state in calculating the magnetic properties of rare-earth manganites. According to the $U_{\text {eff }}$ used in previous studies $\left(U_{\text {eff }}<2\right.$

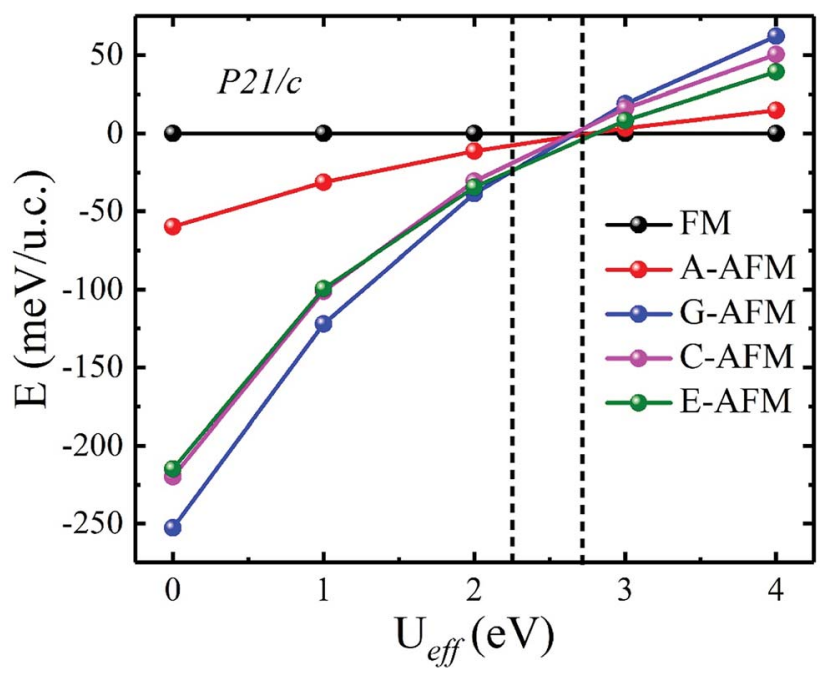

Fig. 5 The calculated phase diagrams of different magnetic orderings and lattice structure parameters as a function of $U_{\text {eff. }}$ The energy difference is defined as $\Delta E=E_{\mathrm{FM}}-E_{\mathrm{AFM}}$. The vertical dashed lines represent the phase boundaries between different magnetic orderings.

$\mathrm{eV})$, $^{\mathbf{4 3 4 4 , 5 0 , 5 1}}$ the magnetic ground state of PSMO is G-AFM. In order to overcome the problems related to the arbitrary choice of the Hubbard $U$ parameter, we also carry out total energy calculations within the HSE06 function for PSMO with different magnetic orderings. ${ }^{46}$ The calculated results also show that the G-AFM state has the lowest energy (see Table 1).

\section{Conclusion}

In conclusion, the lattice, electric, and magnetic properties of highly distorted monoclinic PSMO have been systematically investigated with first-principles calculations. We find that the magnetic ground state of PSMO is the G-AFM state. It is also found that the G-AFM state can give correct structural information in describing the displacements of $\mathrm{Mn}^{3+}$ ions. Moreover, an unreported three-dimensional alternating cooperative orbital ordering is also reported in this study. Because of the smaller bond angle of $<\mathrm{Mn}-\mathrm{O}-\mathrm{Mn}$ induced by the high JT distortion, the nearest-neighbor $\mathrm{Mn}^{3+}$ ions exhibit an AFM magnetic interaction, although PSMO has a three-dimensional cooperative orbital ordering. Moreover, it is found that the PSMO is a bipolar antiferromagnetic semiconductor in which completely spin-polarized currents with reversible spin polarization can be tuned simply by applying a gate voltage. This controllability of the spin polarization of the current opens up new avenues for future spintronic devices. Our calculated results not only suggest that the ground magnetic ordering of PSMO is the G-AFM state, but also enrich the study of orbital ordering in rare-earth manganites. It is expected that more meticulous experiments detecting the magnetic structure of PSMO will validate our theoretical study and complete the magnetic phase diagram of rare-earth manganites. 


\section{Conflicts of interest}

There are no conflicts to declare.

\section{Acknowledgements}

This work has been supported by the National Natural Science Foundation of China though Grant No. 11604113. We are grateful to the High Performance Computing Center (HPCC) of Nanjing University for doing the numerical calculations in this paper on its blade cluster system.

\section{References}

1 E. Dagotto, T. Hotta and A. Moreo, Phys. Rep., 2001, 344, 1153.

2 B. Lorenz, Y.-Q. Wang and C.-W. Chu, Phys. Rev. B: Condens. Matter Mater. Phys., 2007, 76, 104405.

3 D. Lee, A. Yoon, S. Y. Jang, J. G. Yoon, J. S. Chung, M. Kim, J. F. Scott and T. W. Noh, Phys. Rev. Lett., 2011, 107, 057602.

4 Y. S. Chai, Y. S. Oh, L. J. Wang, N. Manivannan, S. M. Feng, Y. S. Yang, L. Q. Yan, C. Q. Jin and K. H. Kim, Phys. Rev. B: Condens. Matter Mater. Phys., 2012, 85, 184406.

5 S. Mukherjee, A. Dönni, T. Nakajima, S. Mitsuda, M. Tachibana, H. Kitazawa, V. Pomjakushin, L. Keller, C. Niedermayer, A. Scaramucci and M. Kenzelmann, Phys. Rev. B: Condens. Matter Mater. Phys., 2017, 95, 104412.

6 H. Y. Hwang, S. W. Cheong, P. G. Radaelli, M. Marezio and B. Batlogg, Phys. Rev. Lett., 1995, 75, 914-917.

7 D. A. Mota, A. Almeida, V. H. Rodrigues, M. M. R. Costa, P. Tavares, P. Bouvier, M. Guennou, J. Kreisel and J. A. Moreira, Phys. Rev. B: Condens. Matter Mater. Phys, 2014, 90, 054104.

8 T. Goto, T. Kimura, G. Lawes, A. P. Ramirez and Y. Tokura, Phys. Rev. Lett., 2004, 92, 257201.

9 B. Dabrowski, S. Kolesnik, A. Baszczuk, O. Chmaissem, T. Maxwell and J. Mais, J. Solid State Chem., 2005, 178, 629-637.

10 A. Oleaga, A. Salazar, D. Prabhakaran, J. G. Cheng and J. S. Zhou, Phys. Rev. B: Condens. Matter Mater. Phys., 2012, 85, 184425.

11 J. S. Zhou, J. B. Goodenough, J. M. Gallardo-Amores, E. Morán, M. A. Alario-Franco and R. Caudillo, Phys. Rev. B: Condens. Matter Mater. Phys., 2006, 74, 014422.

12 R. Basistyy, T. N. Stanislavchuk, A. A. Sirenko, A. P. Litvinchuk, M. Kotelyanskii, G. L. Carr, N. Lee, X. Wang and S. W. Cheong, Phys. Rev. B: Condens. Matter Mater. Phys., 2014, 90, 024307.

13 T. Katsufuji, M. Masaki, A. Machida, M. Moritomo, K. Kato, E. Nishibori, M. Takata, M. Sakata, K. Ohoyama, K. Kitazawa and H. Takagi, Phys. Rev. B: Condens. Matter Mater. Phys., 2002, 66, 134434.

14 M. Tachibana, T. Shimoyama, H. Kawaji, T. Atake and E. Takayama-Muromachi, Phys. Rev. B: Condens. Matter Mater. Phys., 2007, 75, 144425.
15 T. Kimura, S. Ishihara, H. Shintani, T. Arima, K. T. Takahashi, K. Ishizaka and Y. Tokura, Phys. Rev. B: Condens. Matter Mater. Phys., 2003, 68, 060403.

16 J. S. Zhou and J. B. Goodenough, Phys. Rev. Lett., 2006, 96, 247202.

17 I. Solovyev, N. Hamada and K. Terakura, Phys. Rev. Lett., 1996, 76, 4825-4828.

18 Y. Murakami, J. P. Hill, D. Gibbs, M. Blume, I. Koyama, M. Tanaka, H. Kawata, T. Arima, Y. Tokura, K. Hirota and Y. Endoh, Phys. Rev. Lett., 1998, 81, 582-585.

19 P. Mandal and S. Das, Phys. Rev. B: Condens. Matter Mater. Phys., 1997, 56, 15073-15080.

20 J. Hemberger, M. Brando, R. Wehn, V. Y. Ivanov, A. A. Mukhin, A. M. Balbashov and A. Loidl, Phys. Rev. B: Condens. Matter Mater. Phys., 2004, 69, 064418.

21 T. Kimura, T. Goto, H. Shintani, K. Ishizaka, T. Arima and Y. Tokura, Nature, 2003, 426, 55-58.

22 M. Kenzelmann, A. B. Harris, S. Jonas, C. Broholm, J. Schefer, S. B. Kim, C. L. Zhang, S. W. Cheong, O. P. Vajk and J. W. Lynn, Phys. Rev. Lett., 2005, 95, 087206.

23 T. Kimura, G. Lawes, T. Goto, Y. Tokura and A. P. Ramirez, Phys. Rev. B: Condens. Matter Mater. Phys., 2005, 71, 224425.

24 O. Prokhnenko, R. Feyerherm, E. Dudzik, S. Landsgesell, N. Aliouane, L. C. Chapon and D. N. Argyriou, Phys. Rev. Lett., 2007, 98, 057206.

25 A. Munoz, M. T. Casais, J. A. Alonso, M. J. Martinez-Lope, J. L. Martinez and M. T. Fernandez-Diaz, Inorg. Chem., 2001, 40, 1020-1028.

26 H. Chen, T. Yu, P. Gao, J. Bai, J. Tao, T. A. Tyson, L. Wang and R. Lalancette, Inorg. Chem., 2013, 52, 9692-9697.

27 T. Yu, T. A. Tyson, H. Y. Chen, A. M. M. Abeykoon, Y. S. Chen and K. H. Ahn, J. Phys.: Condens. Matter, 2014, 26, 495402.

28 V. M. Goldschmidt, Naturwissenschaften, 1926, 14, 477-485.

29 J.-W. G. Bos, B. B. van Aken and T. T. M. Palstra, Chem. Mater., 2001, 13, 4804-4807.

30 P. E. Blöchl, Phys. Rev. B: Condens. Matter Mater. Phys., 1994, 50, 17953-17979.

31 G. Kresse and J. Hafner, Phys. Rev. B: Condens. Matter Mater. Phys., 1993, 47, 558-561.

32 G. Kresse and J. Furthmüller, Phys. Rev. B: Condens. Matter Mater. Phys., 1996, 54, 11169-11186.

33 J. P. Perdew, A. Ruzsinszky, G. I. Csonka, O. A. Vydrov, G. E. Scuseria, L. A. Constantin, X. Zhou and K. Burke, Phys. Rev. Lett., 2008, 100, 136406.

34 J. P. Perdew, K. Burke and M. Ernzerhof, Phys. Rev. Lett., 1996, 77, 3865-3868.

35 J. P. Perdew and A. Zunger, Phys. Rev. B: Condens. Matter Mater. Phys., 1981, 23, 5048-5079.

36 S. L. Dudarev, G. A. Botton, S. Y. Savrasov, C. J. Humphreys and A. P. Sutton, Phys. Rev. B: Condens. Matter Mater. Phys., 1998, 57, 1505-1509.

37 J. Kanamori, J. Appl. Phys., 1960, 31, S14-S23.

38 C. P. Adams, J. W. Lynn, Y. M. Mukovskii, A. A. Arsenov and D. A. Shulyatev, Phys. Rev. Lett., 2000, 85, 3954-3957.

39 Y. S. Hou, H. J. Xiang and X. G. Gong, Phys. Rev. B: Condens. Matter Mater. Phys., 2014, 89, 064415. 
40 G. R. Blake, T. T. M. Palstra, Y. Ren, A. A. Nugroho and A. A. Menovsky, Phys. Rev. Lett., 2001, 87, 245501.

41 J. B. Goodenough, Phys. Rev., 1955, 100, 564-573.

42 J. Kanamori, J. Phys. Chem. Solids, 1959, 10, 87-98.

43 K. Yamauchi, F. Freimuth, S. Blügel and S. Picozzi, Phys. Rev. B: Condens. Matter Mater. Phys., 2008, 78, 014403.

44 S. Picozzi, K. Yamauchi, G. Bihlmayer and S. Blügel, Phys. Rev. B: Condens. Matter Mater. Phys., 2006, 74, 094402.

45 J. C. Slater and G. F. Koster, Phys. Rev., 1954, 94, 1498-1524.

46 J. Heyd, J. E. Peralta, G. E. Scuseria and R. L. Martin, J. Chem. Phys., 2005, 123, 174101.
47 X. Li, X. Wu, Z. Li, J. Yang and J. G. Hou, Nanoscale, 2012, 4, 5680-5685.

48 I. Solovyev, N. Hamada and K. Terakura, Phys. Rev. B: Condens. Matter Mater. Phys., 1996, 53, 7158-7170.

49 I. A. Vladimir, F. Aryasetiawan and A. I. Lichtenstein, J. Phys.: Condens. Matter, 1997, 9, 767.

50 G. Li, X. Huang, J. Hu, G. Song and W. Zhang, Phys. Rev. B: Condens. Matter Mater. Phys., 2018, 97, 085140.

51 J. T. Zhang, C. Ji, J. L. Wang, W. S. Xia, X. M. Lu and J. S. Zhu, Phys. Rev. B: Condens. Matter Mater. Phys., 2018, 97, 085124. 52 I. Solovyev, J. Phys. Soc. Jpn., 2009, 78, 054710. 